\title{
A Multi-Modal Intervention To Improve Hand Hygiene in The Health Care Facility and Home Environment During The Peri-Natal Period in Kampong Chhnang, Cambodia: A Controlled Before-And-After Feasibility Study
}

\author{
Yolisa Nalule ( $\sim$ Yolisa.Nalule@lshtm.ac.uk) \\ London School of Hygiene \& Tropical Medicine \\ Ponnary Pors \\ WaterAid \\ Channa Samol \\ WaterAid \\ Senghort Ret \\ WaterAid \\ Supheap Leang \\ National Institute of Public Health \\ Por Ir \\ National Institute of Public Health \\ Alison Macintyre \\ WaterAid

\section{Robert Dreibelbis} \\ London School of Hygiene \& Tropical Medicine
}

\section{Research Article}

Keywords: Hand hygiene, Maternal health, Newborn health

Posted Date: October 25th, 2021

DOI: https://doi.org/10.21203/rs.3.rs-971007/v1

License: (c) (1) This work is licensed under a Creative Commons Attribution 4.0 International License. Read Full License 


\section{Abstract \\ Background}

Adequate hand hygiene practices throughout the continuum of care of maternal and newborn health are essential for infection prevention. However, the hand hygiene compliance of facility-based birth attendants, parents and other caregivers along this continuum is low and behavioural-science informed interventions targeting the range of caregivers in both the healthcare facility and home environments are scarce. We assessed the limited efficacy of a novel multimodal behaviour change intervention, delivered at the facility, to improve the hand hygiene practices among midwives and caregivers during childbirth through the return to the home environment.

\section{Methods}

The 6-month intervention was implemented in 4 of 8 purposively selected facilities and included environmental restructuring, hand hygiene infrastructure provision, cues and reminders, and participatory training. In this controlled before-and-after study, the hand hygiene practices of all caregivers present along the care continuum of 99 women and newborns were directly observed. Direct observations took place during three time periods; labour, delivery and immediate aftercare in the facility delivery room, postnatal care in the facility ward and in the home environment within the first 48hrs following discharge. Multilevel logistic regression models, adjusted for baseline measures, assessed differences in hand hygiene practices between intervention and control facilities.

\section{Results}

The intervention was associated with increased odds of improved practice of birth attendants during birth and newborn care in the delivery room (Adjusted odds ratio $[\mathrm{AOR}]=4.7 ; 95 \%$ confidence interval $[\mathrm{Cl}]=2.7,7.7$ ), and that of parental and non-parental caregivers prior to newborn care in the post-natal care ward $(\mathrm{AOR}=9.2 ; \mathrm{Cl}=1.3,66.2)$; however, the absolute magnitude of improvements was limited. Intervention effects were not presented for the home environment due COVID-19 related restrictions on observation duration at endline which resulted in too low observation numbers to warrant testing.

\section{Conclusion}

Our results suggest the potential of a facility-based multimodal behaviour change intervention to improve hand hygiene practices that are critical to maternal and neonatal infection along the continuum of care.

\section{Background}

Globally, infections acquired during birth and the first days of post-natal care account for an estimated 11- 19\% of all neonatal deaths (1) and $10 \%$ of maternal deaths (2), the majority of which occur in low and middle income countries (LMIC). Adequate hand hygiene practices are an essential maternal and newborn infection prevention strategy (3-6) and should be delivered throughout the continuum of care (7-10). The continuum of care for maternal, newborn and child health is typically defined to include care during pregnancy, delivery and postnatal care (PNC) $(11,12)$. 
Hand hygiene compliance of birth attendants and caregivers along this care continuum remains low (13-16). A recent systematic review of facility-based birth attendants' hand hygiene compliance in LMIC estimated compliance ranging from 1-38\% (13). Observational studies of facility-based births in LMIC have identified multiple risks of infection to mothers and newborns due to inadequate hand hygiene practices during post-natal care in the facility through to the return to the household (14-19).

Hygiene interventions spanning both healthcare facility (HCF) and home environments are scarce with limited evidence around their effectiveness at changing handwashing behaviour $(11,20)$. Hand hygiene studies to-date mostly focus on individual care periods, particularly birth $(13,17,18,21-23)$ or the late post-natal period $(>7$ days of life) (24-27). Postnatal care in the critical days following facility-based birth remains under investigated (11, 12). The role and engagement of paternal and non-parental caregivers in LMIC in the provision of care across both environments has been documented $(15,19,24,25,28,29)$, however hand hygiene improvement strategies commonly only target birth attendants and mothers' practices, leaving other important caregiver groups' practices unaddressed (20). There is a need for contextualised hand hygiene strategies that can span both the HCF and the home and effectively target hand hygiene practices of the multiple caregivers that lie along this pathway.

We tested the feasibility of a facility-based intervention targeting maternal and newborn caregivers' hand hygiene practices during institutional births and post-natal care, and the return to the home environment. Formative work in this setting documented low hand hygiene compliance in both the facilities and in the home and identified various psychosocial, physical and contextual factors influencing these practices $(18,19)$. Despite availability of functioning hand hygiene infrastructure and materials and demonstrated understanding of infection risk due to inadequate hand hygiene, barriers to effective hand hygiene adherence from midwives included limited understanding of hand or glove recontamination risk, habits and norms around inappropriate gloving practices, perceived lack of time from high workloads and low staffing, and low perception of newborn infection risk (18). Among household members, barriers to effective hand hygiene included inadequate and inaccessible hygiene infrastructure in the facility, inadequate knowledge around hand hygiene opportunities and adequate hygiene protocol during newborn care, bypassing handwashing steps due to excitement or rushing to alleviate the newborn's perceived distress (nurture), and restrictive gender norms and family hierarchies resulting in limited influence of the mother to inform other relatives' hand hygiene practices (19).

These findings informed the design of a multimodal behaviour change intervention delivered at facility level and aimed at improving hygiene behaviours related to maternal and neonatal care at both healthcare facilities and the home environment. The objective of this controlled before and after study was to assess the effect of this intervention on hand hygiene behaviours among birth attendants and other caregivers across eight HCFs in rural Cambodia.

\section{Methods}

\section{Study setting and participants}

The study was conducted in Kampong Chhnang Province, Cambodia. The study included eight purposively selected healthcare facilities (HCF), six primary health centres (PHC) and two referral hospitals, that were initially observed in the formative phase of the project $(18,19)$. Four HCFs were assigned to receive the intervention and the remaining four assigned to serve as comparison facilities. The six participating PHCs represented three different settings; rural/low facility-birth volume, rural/high facility-birth volume, and peri-urban/high facility-birth volume. One facility from each setting was assigned to receive the intervention. The referral hospital with the 
higher number of monthly deliveries was selected to receive the intervention. In the six PHCs, women were recruited until either five births per facility had been observed or the 14-day observation period ended. In the referral hospitals as many women as possible were recruited and observed over a period of 14 days. The sample size was considered sufficient for the exploratory nature of the study.

Any woman who presented to the HCF for delivery prior to entering the second stage of labour and was not already in excess pain and distress was eligible for recruitment. Patients considered by clinical staff to have complicated labour or delivery, or those under 18 and unaccompanied by a parent/guardian were excluded from the study. Written consent was obtained from the women, health care workers and all accompanying members who were present with the woman at any point during the observation period. We obtained verbal consent in addition to a witness signature in the case of a participant with low literacy. Participation was voluntary and the recruitment was done in a private area and the women were encouraged to have someone else with them during the recruitment process. To minimise reactivity, the explicit mention of handwashing was avoided and participants were informed that the aim of the study was to observe care giving practices during childbirth and postnatal care. The data collector discussed and agreed verbal or non-verbal cues with each participant that they could use to pause or terminate the observation at any time.

\section{Intervention package}

The design and development of the intervention was informed by earlier formative research conducted in the 8 HCFs $(18,19)$. The Behaviour Centred Design (BCD) theoretical framework was used to guide the collection and analysis of the formative research data (30). Following the eco - evo theory understanding of human behaviours, the BCD approach provides a systematic means of categorising behavioural determinants and translate these determinants into specific intervention components.

Potential interventions were tested and refined through a participatory creative process led by 17 Triggers, an incountry creative agency. Following a three-day co-creation workshop with key stakeholders in Phnom Penh, rapid field testing and prototyping was conducted with 15 mothers, 10 midwives, two facility directors and seven family members in two non-study HCFs and catchment communities over three weeks in November 2019. The final intervention design was based on the level of acceptability by users and HCF management, theoretical considerations and logistic and financial constraints. The final intervention was a multimodal intervention targeting midwives, mothers, fathers and non-parental caregivers that included physical environment restructuring, provision and improved access to hand hygiene infrastructure and materials, visual cues and reminders, social influence, and participatory training.

The intervention was delivered in two locations within the maternity ward; the labour and delivery (LD) room and PNC room. The intervention components in the LD room aimed to improve hand hygiene practices of birth attendants, primarily midwives, during birth and the intervention components in the PNC room aimed to improve the hand hygiene practices of primarily mothers and caregivers providing early newborn care in both the post-natal care ward and the household following discharge. Table 1 provides an overview of the intervention components and content. 
Table 1

Overview of intervention components and content

Intervention Component Content and purpose
location

\section{Labour and Delivery room}

A set of bright coloured hand hygiene icon stickers strategically placed by midwives at key points in the room where hand hygiene opportunities most often occurred during birth.

Painting the labour and delivery hand washing station area and installing a similarly coloured soap dispenser to visibly link the reminder to the required practice

\section{Social influence A group commitment/pledge made by midwives to always practice proper} hand hygiene and hold each other accountable

Hand hygiene badges for midwives to wear on their uniforms meant to encourage midwives to ask each other about their hand washing activities at key points during their shifts.

A token jar and counters placed in a visible area for midwives to tally how often they reminded each other per week

\section{Participatory} hygiene training

The training was conducted in the labour room and delivered digitally/remotely by a facilitator from the creative agency with experience in both participatory techniques and overseen by a technical lead from National Institute of Public Health - Cambodia (NIPH) and WaterAid. Midwives were trained using a combination participatory group discussions, creative exercises, scripts and role play. All training was based on Ministry of Health and WHO guidelines for technical validity.

Midwives training aimed to improve and refresh knowledge on adequate hygiene protocol specific to LD events (labour, delivery and immediate newborn care) including identifying recontamination events and the corresponding hand hygiene protocol.

With the exception of the painting, all labour and delivery room intervention components were delivered and set up by the midwives as part of the training.

\section{Post-natal} care ward
Visual demarcation of the post-natal care ward as a "clean hands" zone by painting the ward door and wall section bright green

Placing hand hygiene icon stickers illustrating proper hand hygiene technique and nurture-evoking hygiene messaging posters across all postnatal care ward hand hygiene infrastructure and toilets

Provision of a brightly coloured hat to each baby with visible hygiene-related messaging
Installation of a matching coloured hand washing station, liquid soap dispensers and soap at the ward entrance

Installation of matching coloured alcohol-based handrub station at the end of each bed

Provision of personal alcohol-based handrub bottle for each mother 


\begin{tabular}{|c|c|c|}
\hline $\begin{array}{l}\text { Intervention } \\
\text { location }\end{array}$ & Component & Content and purpose \\
\hline & \multirow[t]{2}{*}{$\begin{array}{l}\text { Behaviour } \\
\text { Change } \\
\text { Communication }\end{array}$} & $\begin{array}{l}\text { Newborn hygiene - related information would be passed on to mother along } \\
\text { with the personal alcohol-based handrub bottle and baby hat during routine } \\
\text { care advice in the labour and delivery room following birth }\end{array}$ \\
\hline & & $\begin{array}{l}\text { Newborn hygiene - related information would be passed on to the present } \\
\text { visitors during the routine post-natal care advice given upon admission of } \\
\text { the mother-newborn pair to the post-natal care ward. The 'clean hands' zone } \\
\text { concept was to be explained to the visitors at this time or earlier during } \\
\text { routine cervical checks when the admitted mother and caregivers were in } \\
\text { PNC ward during stage } 1 \text { labour. }\end{array}$ \\
\hline & \multirow[t]{4}{*}{$\begin{array}{l}\text { Participatory } \\
\text { hygiene training }\end{array}$} & $\begin{array}{l}\text { Similar to LD training component, the training was delivered digitally to the } \\
\text { midwives via participatory group discussions, creative exercises, scripts and } \\
\text { role play in the post-natal care ward. All training was based on National } \\
\text { Ministry of Health and WHO guidelines for technical validity }\end{array}$ \\
\hline & & Training included: \\
\hline & & $\begin{array}{l}\text { - Adequate hygiene protocol specific to newborn aftercare for all caregivers } \\
\text { in the ward and at home }\end{array}$ \\
\hline & & $\begin{array}{l}\text { - Familiarisation with the post-natal care ward intervention components } \\
\text { including deciding how and when to introduce the intervention components } \\
\text { and pass on the behaviour change communication to the family members } \\
\text { and mother }\end{array}$ \\
\hline
\end{tabular}

The intervention was delivered to selected facilities in July 2020 and ran for 24 weeks. Throughout the 6-month implementation period, the intervention HCFs were responsible for refilling the liquid soap and maintaining the handwashing sinks. The study project team was responsible for restocking and maintaining all other intervention components. Final data collection ran from November to December 2020.

\section{Study design and evaluation}

\section{Outcomes}

The study was designed as a non-randomised controlled before-and-after feasibility study. The behavioural outcomes of interest were hand hygiene practices of 1) Birth attendants during labour, delivery and immediate newborn care in the delivery room; 2) Health care workers and other caregivers in post-natal care facility ward during newborn care and 3) Caregivers in the home environment during newborn care. Previously reported observational data on hand hygiene $(18,19)$ served as the baseline measures for this evaluation.

\section{Data collection procedures}

Data (baseline) was collected in all eight participating facilities (February - July 2019) as part of the formative research study and were repeated at the end of the implementation period (endline). All study methods, data collection procedures, tools and baseline findings are detailed in earlier publications $(18,19)$. Structured assessments were conducted to assess the facility-level and household hygiene conditions. Hand hygiene practices were assessed via direct observation over three periods; childbirth in the delivery ward, post-natal period in the facility PNC ward and post-natal period in the home. 
Labour and delivery observations began when the woman was admitted to the facility and the first vaginal examination occurred and terminated either after six hours or when the woman-newborn pair was discharged from the delivery room, whichever came first. Data collectors recorded key events including birth attendants' handwashing and gloving practices and any observed contact of the birth attendants with the mother, newborn, objects and surfaces and other individuals during the observation period.

Observations during the post-natal period in the facility began when the mother-newborn pair was transferred to the PNC room of participating facilities and were terminated after a period of four continuous hours. Home observations were conducted within 72 hours following discharge from the HCF and began after the household structured assessment was completed and lasted one hour. Home observations were only completed for women who delivered in the six PHCs. During both the PNC period at the facility and the home, newborn care practices included diaper changes, cord care, breastfeeding and general newborn handling and were recorded along with any corresponding hand hygiene practices of any individuals providing newborn or maternal care.

All women were given a 15-minute break from observations every two hours, but any of the participants could use the agreed verbal or nonverbal cues to pause or terminate the observations at any time.

Data were collected on tablets used pre-coded observation tools by trained data collectors. Across all observations, data collectors positioned themselves in an unobtrusive location and recorded key events of all individuals present in each respective period. Five of the six study observers had prior experience with the study protocol and data collection methods having participated in the baseline data collection. All observers received a 3-day in house refresher training of the study protocol, ethical research practices and role play sessions. All observations tools and protocols were piloted in the field prior to data collection, however due to COVID-19 risk measures, the pilots were conducted in the study referral hospitals where the midwives observed four deliveries. Study observers were paired up during the pilot period to increase interrater reliability. Given the small sample size, rather than use statistical techniques to formally test reliability, observers worked independently to gather data and the observation data was compared at the end of each delivery. Any discrepancies between the pairs were discussed with the study manager for clarification and discussion.

\section{Data analysis}

All quantitative data was analysed using StataSE 15 (Stata Corp, College Station, TX, USA). All Qualitative notes recorded during the observations were reviewed and where applicable, recoded using STATA. Data from the home and facility level assessments were analysed descriptively and triangulated to provide context to the structured observations.

\section{Analysis of Labour and delivery structured observations}

We defined labour, delivery and newborn aftercare flows according to the analysis described in the methods by Nalule et. al (18). For each flow, we assigned time-specific hand hygiene categories (Table 2) to each birth attendant around hand hygiene opportunities prior to the initiation of the flow and within the flow when invalidation of aseptic technique occurred. Detailed definitions and descriptions of flows and aseptic procedures used for the analysis have been previously described (18). 
Table 2

Hand hygiene categories used in analysis

\begin{tabular}{|c|c|c|}
\hline $\begin{array}{l}\text { Observation } \\
\text { period }\end{array}$ & $\begin{array}{l}\text { Hand Hygiene } \\
\text { Category }\end{array}$ & Hand hygiene action \\
\hline \multirow{4}{*}{$\begin{array}{l}\text { Labour and } \\
\text { delivery } \\
\text { (all flows) }\end{array}$} & \multirow[t]{2}{*}{ Adequate } & $\begin{array}{l}\text { Handwashing with soap and new gloves (multiple or single) worn } \\
\text { at each hand hygiene opportunity }\end{array}$ \\
\hline & & $\begin{array}{l}\text { No potential recontamination of gloved and/or washed hands } \\
\text { observed }\end{array}$ \\
\hline & Inadequate & $\begin{array}{l}\text { Gloves (multiple or single) are changed without intermediate } \\
\text { handwashing with soap }\end{array}$ \\
\hline & $\begin{array}{l}\text { Aseptic technique } \\
\text { invalidated }\end{array}$ & $\begin{array}{l}\text { No hand hygiene actions taken at observed hand hygiene } \\
\text { opportunity }\end{array}$ \\
\hline \multirow{5}{*}{$\begin{array}{l}\text { Post-natal care in } \\
\text { the facility } \\
\text { Household }\end{array}$} & \multirow[t]{2}{*}{ Adequate } & Handwashing with soap and/or use of alcohol-based handrub; \\
\hline & & $\begin{array}{l}\text { Handwashing with soap and glove use for any aseptic/clean } \\
\text { procedures }\end{array}$ \\
\hline & \multirow[t]{3}{*}{ Inadequate } & Handwashing with water only; \\
\hline & & Wearing gloves without intermediate handwashing with soap; \\
\hline & & $\begin{array}{l}\text { No hand hygiene actions taken at observed hand hygiene } \\
\text { opportunity }\end{array}$ \\
\hline
\end{tabular}

Descriptive statistics were used to calculate the frequency and proportion of flows initiated and aseptic procedures within the flows that were conducted under each respective hand hygiene category. Results were provided by data collection rounds (endline, baseline) and study group assignment (intervention, comparison).

Multilevel logistic regression models accounting for clustering at the facility-level were used to calculate differencein-difference (DID) estimates to assess the effect of the intervention between intervention and comparison facilities after adjusting for baseline observations. The outcome measures for this analysis were:

1). Adequate hand hygiene prior to the initiation of a flow or prior to any key event related to newborn care (coded as adequate hand hygiene category $=1$; aseptic technique invalidated or inadequate hand hygiene categories $=0$ ) 2). Invalidated hand hygiene prior to the initiation of a flow (coded as aseptic technique invalidated hand hygiene category $=1$; inadequate or adequate hand hygiene categories $=0$ ).

3). Adequate hand hygiene during aseptic procedures within the flow (coded as adequate hand hygiene category = 1 ; aseptic technique invalidated or inadequate hand hygiene categories $=0$ )

We ran models separately for each outcome measure. The first level of DID models assessed the differences in outcomes without adjustment for any potential confounding variables. The second set of DID models for outcome measures 1 and 2 adjusted for working shift time, facility type (referral hospital vs primary health facility) and professional qualification (midwife vs Doctor + Nurse vs Midwife intern) and for outcome measures 3 adjusted for facility type (referral hospital vs primary health facility) and professional qualification (midwife vs Doctor + Nurse vs Midwife intern). We present the effect estimates from the DID analyses as odds ratios.

\section{Analysis of PNC and home observation structured observations}


Only hand hygiene opportunities pertaining to newborn care were analysed for the PNC and home observations. Detailed descriptions of newborn-care related hand hygiene opportunities used for this analysis are previously described (19). Caregivers of the newborns were categorised into four groups; mothers, fathers, healthcare workers (midwives, nurses, doctors and interns) or non-parental caregivers (all other individuals observed providing care to the newborn). For each caregiver, hand hygiene actions associated with each hand hygiene opportunity were coded into two categories for the analysis; adequate and inadequate (Table 2).

Descriptive statistics were used to calculate the frequency and proportion of hand hygiene opportunities under each respective hand hygiene category by caregiver and treatment group at endline only. For the PNC in the facility observation period, we ran multilevel logistic regression models; unadjusted and adjusted for working shift time, facility type (referral hospital vs primary health facility) and professional qualification (midwife vs Doctor + Nurse vs Midwife intern). Due to low rates of observed hand hygiene measures at baseline (19), we based our estimate of effect on endline observations only. For home observations, the limited numbers of observations and zero baseline hand hygiene compliance (19) did not warrant significance testing.

\section{Results}

Baseline data were collected in February - July 2019 and endline data in November - December 2020 (Table 3).

\section{Facility and household hygiene conditions}

The delivery room hygiene conditions were similar between study groups at both baseline and endline (Table 3). 
Table 3

Facility and household hygiene conditions

Endline
Baseline

FACILITY

Handwashing stations in delivery

room

Available

Soap and water/Alcohol-based handrub available
Comparison (n

$=4$ )

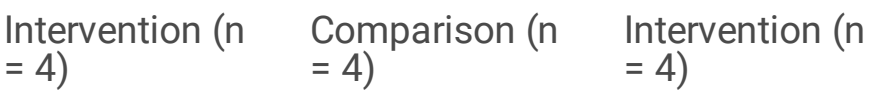

\section{Handwashing facilities inside PNC}

room

Available

Soap and water/Alcohol-based

handrub available

Hand hygiene posters

Delivery room

PNC room

\section{Handwashing station at toilet}

Available
Soap and water/Alcohol-based
handrub available

HOUSEHOLD

Handwashing facility

Available

Soap/detergent available at site
Hand hygiene items

Soap available

Detergent available

Alcohol-based handrub available

\section{Location of Alcohol-based handrub}

Near woman

In kitchen

With household member

$\begin{array}{llll}4(100) & 4(100) & 4(100) & 4(100) \\ 4(100) & 4(100) & 4(100) & 4(100)\end{array}$

$4(100)$

4 (100) $\begin{array}{ll}\text { Intervention ( } & \begin{array}{ll}\text { Comparison ( } & \text { Inter } \\ =4\end{array}\end{array}$

$\begin{array}{ll}\text { Intervention ( } & \begin{array}{ll}\text { Comparison ( } & \text { Inter } \\ =4\end{array}\end{array}$ 
All delivery rooms had functional handwashing facilities (HWF). In the PNC room, none of the facilities had functional HWF or hand hygiene messaging at baseline. At endline, functional HWF were available in 100\% (4/4) of the PNC rooms of the intervention facilities and $0 \%$ of comparison facilities. Hand hygiene messaging was present in $100 \%$ (4/4) of intervention and $25 \%$ (1/4) of comparison facilities.

At baseline, functioning HWF were present in 45\% (5/11) of the households of the women who had given birth at the comparison facilities (comparison households) and $54 \%(6 / 11)$ of the households of the women who had given birth at the intervention facilities (intervention households). The presence of functioning HWF dropped to $9 \%$ $(1 / 11)$ in the comparison households and increased to $73 \%(8 / 11)$ in the intervention households at endline. Over $90 \%$ of the households in both groups had soap and detergent available in the household at both endline and baseline. At endline, ABHR was present was in 92\% (9/11) of the intervention households and $9 \%(1 / 11)$ of the comparison households. All ABHR present in the intervention households was from the facility.

\section{Participant characteristics}

At baseline, 45 mothers ( $n=16$ comparison; $n=29$ intervention) were enrolled and observed during the labour and delivery observations, 46 ( $n=17$ comparison; $n=29$ intervention) during facility PNC observations and 22 ( $n=11$ comparison; $\mathrm{n}=11$ intervention) during the household observations. These details can be found in the supplementary tables provided (see Supplementary Table S1). At endline, 54 mothers ( $n=19$ comparison; $n=35$ intervention) were observed during the labour and delivery observations, 53 ( $n=18$ comparison; $n=35$ intervention) during facility PNC observations and 22 ( $n=11$ comparison; $n=11$ intervention) during the household observations.

Baseline and endline characteristics of the mothers were similar between study facilities although there were some differences in working shift times at endline, number of PNC visitors at baseline and HCF staff presence in both time periods. More details showing the baseline and endline characteristics by intervention group and observation period can be found in the supplementary table (see Supplementary Table S1).

Only one additional participant was recruited for post-natal observations at baseline. At endline, one observation had to be terminated at endline due to newborn complications in the facility PNC ward.

\section{Structured observations in the delivery room}

\section{All flows}

When combined, the proportion of flows initiated under adequate hand hygiene declined by 10\% (24-14\%) in comparison facilities and increased by $11 \%$ (16-27\%) in intervention facilities between baseline and endline (Table 4). After adjusting for baseline differences and potential confounders, this corresponds to an adjusted odds ratio (AOR) of 4.7 (95\% confidence interval $(\mathrm{Cl})=2.9,7.7)$. 
Table 4

Effect of the intervention on adequate hand hygiene during labour and delivery

\begin{tabular}{|c|c|c|c|c|c|}
\hline & $\begin{array}{l}\text { Baseline } \\
\mathbf{N}(\%)\end{array}$ & $\begin{array}{l}\text { Endline } \\
\mathrm{N}(\%)\end{array}$ & $\begin{array}{l}\text { Percentage point } \\
\text { difference (\%) }\end{array}$ & $\begin{array}{l}\mathrm{OR}^{1}(95 \% \\
\mathrm{Cl})\end{array}$ & $\begin{array}{l}\mathrm{AOR}^{2}(95 \% \\
\mathrm{Cl})\end{array}$ \\
\hline $\begin{array}{l}\text { All flows } \\
\text { Intervention }\end{array}$ & $26(16)$ & $69(27)$ & +11 & $\begin{array}{l}3.9(2.3, \\
6.4)\end{array}$ & $\begin{array}{l}4.7(2.9 \\
7.7)\end{array}$ \\
\hline Comparison & $21(24)$ & $17(14)$ & -10 & 1.00 (Ref) & 1.00 (Ref) \\
\hline $\begin{array}{l}\text { Labour } \\
\text { Intervention }\end{array}$ & $14(20)$ & $33(45)$ & +25 & $\begin{array}{l}7.0(1.4 \\
35.3)\end{array}$ & $\begin{array}{l}6.6(1.4 \\
32.1)\end{array}$ \\
\hline Comparison & $8(31)$ & $6(17)$ & -14 & 1.00 (Ref) & 1.00 (Ref) \\
\hline $\begin{array}{l}\text { Delivery }{ }^{5} \\
\text { Intervention }\end{array}$ & $11(17)$ & $16(20)$ & +3 & $\begin{array}{l}\text { 1.8 }(0.7 \\
5.1)\end{array}$ & $\begin{array}{l}1.8(0.5 \\
6.5)\end{array}$ \\
\hline Comparison & $10(26)$ & 7 (19) & -7 & 1.00 (Ref) & 1.00 (Ref) \\
\hline $\begin{array}{l}\text { Newborn Aftercare } \\
\text { Intervention }\end{array}$ & $2(6)$ & $20(20)$ & +14 & $\begin{array}{l}6.3(1.0 \\
38.5)\end{array}$ & $\begin{array}{l}9.7(1.2, \\
75.7)\end{array}$ \\
\hline Comparison & $3(13)$ & $4(8)$ & -5 & 1.00 (Ref) & 1.00 (Ref) \\
\hline $\begin{array}{l}\text { Aseptic events within delivery } \\
\text { flow }{ }^{7} \\
\text { Intervention }\end{array}$ & $26(11)$ & 39 (15) & +4 & $\begin{array}{l}2.4(0.9 \\
5.9)\end{array}$ & $\begin{array}{l}2.6(0.9 \\
7.6)\end{array}$ \\
\hline Comparison & $21(19)$ & $16(12)$ & -7 & 1.00 (Ref) & 1.00 (Ref) \\
\hline $\begin{array}{l}\text { Note. } \text { AOR }=\text { Adjusted Odds Ra } \\
{ }^{1} \text { Clustered by facility }{ }^{2} \text { Adjust } \\
\text { professional qualification (mic } \\
{ }^{4} \text { Baseline } n=95, \text { Endline } n=1 \\
{ }^{7} \text { Baseline } n=340, \text { Endline }=39\end{array}$ & $\begin{array}{l}\text { OR }=\text { Odc } \\
\text { for workin } \\
\text { ife vs Doct } \\
5 \text { Baselin }\end{array}$ & $\begin{array}{l}\text { Ratio } \\
\text { shift tin } \\
+ \text { Nurse } \\
n=102,\end{array}$ & $\begin{array}{l}\text { acility type (refen } \\
\text { Midwife intern); } \\
\text { lline } n=116 ;{ }^{6} \mathrm{Ba}\end{array}$ & $\begin{array}{l}\text { vs primar } \\
=251, \text { En } \\
54, \text { Endline }\end{array}$ & $\begin{array}{l}\text { alth facility), } \\
\text { en = 375; } \\
151\end{array}$ \\
\hline
\end{tabular}

\section{Labour flow}

At endline, $45 \%$ of labour flows in intervention facilities were initiated by the healthcare worker with hands washed and clean gloves worn compared to $17 \%$ in the comparison facilities (Table 4). The proportion of labour flows initiated under adequate hygiene in the comparison facilities declined by $14 \%$ between baseline and endline and increased by $25 \%$ in the intervention facilities. After adjusting for baseline measures and potential confounders, midwives in intervention facilities had almost 7 times greater odds of practicing adequate hand hygiene prior to initiating a labour flow than midwives in comparison facilities $(\mathrm{AOR}=6.6 ; \mathrm{Cl}=1.4,32.1)$.

\section{Delivery flow}


Between baseline and endline, the proportions of delivery flows initiated under adequate hand hygiene increased in the intervention facilities (17-20\%) and declined in the comparison facilities (26-19\%) (Table 4). We found no evidence of difference in the odds of birth attendants initiating delivery flows with adequate hand hygiene between the intervention and comparison facilities $(\mathrm{AOR}=1.8 ; \mathrm{Cl}=0.5,6.5)$.

\section{Hygiene within the delivery flow}

Within the delivery flows, we observed 340 ( $n=108$ comparison; $n=232$ intervention) unique aseptic events at baseline and 398 ( $n=131$ comparison; $n=267$ intervention) at endline. The frequency and proportion of individual aseptic events in detail can be found in the supplementary information (see Supplementary Table S2) shows the. At baseline, $19 \%$ and $11 \%$ of aseptic events within the delivery flow were conducted under adequate hand hygiene in comparison and intervention facilities (Table 4). This proportion declined to $12 \%$ in comparison facilities and increased by 3 percentage points to $15 \%$ in the intervention facilities. We found no evidence of differences in the odds of conducting any aseptic procedure within the delivery flow under adequate hygiene between the intervention and comparison group after adjusting for baseline observation and potential confounders $(A O R=2.6$; $\mathrm{Cl}=0.9,7.6$ ). More details on the proportion of individual aseptic events conducted under adequate hand hygiene can be found in the supplementary information (see Supplementary Table S3)

\section{Newborn aftercare flow}

The proportion of newborn aftercare flows initiated under adequate hand hygiene declined from $13-8 \%$ in the comparison facilities and increased from 6-20\% in intervention facilities between baseline and endline (Table 4). Midwives in an intervention facility had 10 times the odds of practicing adequate hand hygiene prior to initiating a newborn aftercare flow than midwives in the comparison facilities after adjusting for baseline observations and potential confounders $(\mathrm{AOR}=9.7 ; \mathrm{Cl}=1.2,75.7)$.

\section{Invalidated hand hygiene}

We calculated the odds of initiating each of the flow categories under invalidated hand hygiene, coded as invalidated aseptic technique category, compared with either adequate or inadequate hand hygiene categories (Table 2).

The adjusted odds of initiating delivery flow were $70 \%$ lower $(\mathrm{AOR}=0.3, \mathrm{Cl}=0.1-0.7)$ and newborn aftercare flow $60 \%$ lower $(A O R=0.4 ; \mathrm{Cl}=0.2,0.9)$ for midwives in intervention facilities after adjusting for baseline observations and potential confounders. There was no evidence of differences in the odds of initiating the labour flow (AOR = $1.5 ; \mathrm{Cl}=0.7,3.4)$ with invalidated hand hygiene between intervention and comparison facilities. All details of this can be found in the supplementary information (see Supplementary Table S4).

\section{Structured observations in the PNC ward}

At endline, the four-hour observation period began an average of $1.6 \mathrm{hrs}$ (range: 1 - 3) in comparison facilities and $1.1 \mathrm{hrs}(1-3)$ in intervention facilities after the mother-newborn pair was discharged from the LD room (see Supplementary Table S1). The mean number of PNC visitors present during the observations was similar across the groups (4; range 1 - 9). In 39\% ( $n=7)$ of the observations in the comparison facilities, there was no HCW present throughout the 4-hour observation period. Within the intervention facilities only, the installed sinks were functional in $94 \%(n=33)$ of the observations and ABHR stations were functional $100 \%(n=35)$ of the 
observations. $80 \%(n=28)$ of the mothers had their personal ABHR and $86 \%(n=29)$ of the newborns were wearing their intervention hat.

A total of 659 hand hygiene opportunities during newborn care were observed in the PNC ward at endline; $30 \%$ ( $n=$ $198)$ in the comparison group and $70 \%(n=461)$ in the intervention group (Table 5).

Table 5

Effect of the intervention on behavioural outcomes during post-natal care in ward and household

\begin{tabular}{|c|c|c|c|c|c|c|}
\hline & \multicolumn{2}{|c|}{$\begin{array}{l}\text { Hand hygiene opportunities } \\
\mathrm{N}(\%)\end{array}$} & \multicolumn{2}{|c|}{$\begin{array}{l}\text { Adequate hand } \\
\text { hygiene } \\
\mathrm{N}(\%)\end{array}$} & \multirow[b]{2}{*}{$\begin{array}{l}\text { Odds } \\
\text { Ratio } \\
(95 \% \\
\mathrm{Cl})^{1}\end{array}$} & \multirow[b]{2}{*}{$\begin{array}{l}\text { Adjusted } \\
\text { Odds ratio } \\
(95 \% \mathrm{Cl})^{2}\end{array}$} \\
\hline & Comparison & Intervention & Comparison & Intervention & & \\
\hline \multicolumn{7}{|l|}{$\begin{array}{l}\text { Post-natal } \\
\text { care ward }\end{array}$} \\
\hline $\mathrm{HCW}$ & $7(4)$ & $11(2)$ & $0(0)$ & $0(0)$ & & \\
\hline Mothers & $26(13)$ & $42(9)$ & $0(0)$ & $5(12)$ & & \\
\hline Fathers & $30(15)$ & $88(19)$ & $0(0)$ & $10(11)$ & & \\
\hline $\begin{array}{l}\text { Non- } \\
\text { parental } \\
\text { caregivers }\end{array}$ & $135(68)$ & $320(70)$ & $2(2)$ & $27(8)$ & & \\
\hline Total & $198(100)$ & $461(100)$ & $2(1)$ & $42(9)$ & $\begin{array}{l}9.8 \\
(1.5 \\
63.9)^{3}\end{array}$ & $\begin{array}{l}9.2(1.3 \\
66.2)^{3}\end{array}$ \\
\hline \multicolumn{7}{|c|}{$\begin{array}{l}\text { Home } \\
\text { environment }\end{array}$} \\
\hline Mothers & $56(41)$ & $51(41)$ & $0(0)$ & $1(2)$ & & \\
\hline Fathers & $10(7)$ & $6(5)$ & $0(0)$ & $0(0)$ & & \\
\hline $\begin{array}{l}\text { Non- } \\
\text { parental } \\
\text { caregivers }\end{array}$ & $71(52)$ & $68(54)$ & $0(0)$ & $2(3)$ & & \\
\hline Total & $137(100)$ & $125(100)$ & $0(0)$ & $3(2)$ & & \\
\hline
\end{tabular}

9\% (42/462) of all hand hygiene opportunities were met with adequate hand hygiene practice in the intervention group by all caregiver groups except the health care workers. In the comparison group, only $1 \%(2 / 198)$ of all hand hygiene opportunities were conducted under adequate hand hygiene practice all of which were conducted by non- 
parental caregivers. After adjusting for shift time, facility type and professional qualification, caregivers in the intervention group had 9 times the odds of practicing adequate hygiene during newborn care than those in comparison group $(\mathrm{AOR}=9.2 ; \mathrm{Cl}=1.3-66.2)$.

\section{Structured observations in the home}

In the home environment, the one-hour observation period took place the same day the mother-newborn pair was discharged across both study groups. The mean number of non-maternal caregivers present at home was similar and ranged from 2 - 11 in the comparison households and 3-12 in the intervention households.

A total of 262 hand hygiene opportunities were observed at home; 137 (52\%) in the comparison households and 125 (48\%) in the intervention households (Table 5). Non-parental caregivers accounted for over half of the hand hygiene opportunities in both comparison and intervention households. Only $2 \%(3 / 125)$ of the hand hygiene opportunities were conducted under adequate hand hygiene in the intervention households. No adequate hand hygiene was observed being conducted across the caregivers in the comparison households.

\section{Discussion}

Our analyses show the potential impact of a facility-based intervention to improve hand hygiene practices among birth attendants and other caregivers during childbirth and early post-natal care in the healthcare facility environment. Compared to baseline observations and comparison facilities, birth attendants' hand hygiene in the delivery ward improved throughout the childbirth process (all flows combined), and in particular prior to initiating maternal care during early and active labour and initiating newborn care during the 1 st hour of life. The intervention was also effective in increasing adequate hand hygiene practices of mothers, fathers and non-parental caregivers prior to newborn care in the post-natal care room of the facility.

Similar to wider literature on hygiene practices in $\operatorname{HCF}(16,31-36)$ and domestic settings $(7,8,16,24,32)$, hand hygiene practices in our context were influenced by several factors and performed by various people $(18,19)$. Our intervention addressed multiple identified determinants of a wide range of relevant target groups which may explain its apparent success $(13,34,37-40)$. Our findings are in line with multiple systematic reviews showing that interventions to improve hand hygiene behaviours are more effective when they target the context and behaviourspecific determinants of hand hygiene (37-39). Our intervention facilitated and reinforced handwashing practice of target groups through the creation of an enabling environment (physical and social), the incorporation of several nudges \& cues and leveraging the teachable moments of pregnancy/new parenthoods and facility attendance. A detailed process evaluation exploring the factors associated with the intervention success and their potential mechanisms of change is under-development.

Our intervention was successful at reducing the odds of birth attendants initiating a delivery flow with invalidated aseptic technique. However, the odds of initiating delivery flows following full hygiene protocol (hands washed with soap AND glove change) did not change. This result suggests that suboptimal hand hygiene practice, specifically glove changing without intermediate hand washing with soap, was a common occurrence in the facilities. This finding is consistent with other studies in other health care settings $(38,41-45)$ and specifically during delivery $(18,31,35,36,46)$ that have identified institutional factors (human resource shortages, high workload) as influencing the inadequate practice of gloving as a timesaving hand hygiene substitute. Due to 
limited study scope and funding, our intervention did not directly address factors at the institution level which may explain its limited effectiveness at changing gloving practices (46).

Our findings suggest an improvement in hand hygiene compliance after events that invalidated aseptic technique, however confidence intervals overlapped the null value. Despite the persistent challenge avoiding glove/hand recontamination presents to infection control efforts in both $\operatorname{HIC}(47-49)$ and $\operatorname{LMIC}(17,35,50)$, its contribution to overall HCW hand hygiene compliance and subsequently nosocomial transmissions remains under investigated $(17,47,51)$. In labour wards in Tanzania, Gon and colleagues (17) found that aseptic technique was invalidated almost immediately after gloving or hand hygiene action approximately half the time (227/501) A qualitative study by Hor et al (47) across two hospitals in Australia documented frequent risk of hand and glove recontamination during HCW team ward rounds. Further research is warranted to establish the frequency of aseptic invalidation in health care settings and understand the corresponding behavioural determinants to adapt current hand hygiene interventions to specifically address these behaviours.

Overall we observed large relative estimates of effect of the intervention among HCW and household members in the facility; however, the absolute magnitude of change was limited. Additionally, our intervention resulted in significant improvements in hand hygiene of parental and non-parental caregivers in the intervention group but the improved hand hygiene compliance was still very low (<10\%). Linking our hand hygiene intervention to the antenatal care (ANC) period may provide more opportunities to reinforce and address barriers to hand hygiene prior to childbirth. A recent systematic review found that that interventions that linked antenatal care (ANC), birth, and post-natal care were more effective in improving health outcomes compared to those with fewer linkages (12).

Our study employed a one-time onsite training to midwives, delivered remotely over 3 days at the start of the 6month intervention. However, multiple targeted, repetitive short on-site training sessions delivered in appropriately spaced time periods focusing on practical skill development and application have been associated with improvements in HCW knowledge and skills competency compared to the traditional concentrated one-time training approaches and has been shown to be effective in multiple LMIC context (52-55).

Our intervention had no effect on improving hand hygiene practices in the home environment. This finding should be interpreted with caution. In alignment with the Royal Government of Cambodia's national COVID guidelines for household data collection, the endline observation period were limited to only one hour (compared to six hours at baseline). The resulting number of observations due to this reduced observation duration period was too low for our study to sufficiently explore hygiene behaviour change across and within study groups during this critical period (56). A longer observation period at the households would allow for sufficient data to adequately analyse our intervention effects (56). Facility-based interventions have shown promising results as a scalable delivery platform for changing water, sanitation and hygiene (WASH) behaviours at the household level (57-60). The HCF is hypothesized to be a uniquely placed setting to trigger behaviour change as patients and visitors are more likely to be receptive to adopting or improving health behaviours due to heightened perceptions of health risks and the benefits of prevention $(61,62)$. The period of pregnancy and early parenthood have also been identified as similar situations with high motivation and receptivity of those affected to improve health behaviours (62-64). Taken together, the time spent at the facility during antenatal care, labour, delivery and post-natal care remains an opportune setting for targeting expectant parents and non-parental caregivers for sustained behaviour change at the household level (56).

\section{Limitations}

Page $16 / 22$ 
The measurement of behaviour could be prone to reactivity during observation. It was not possible to mask the target recipients or data collectors to the study group status of the facilities due to the visibility of the intervention. Differential reactivity between study groups could result in over- estimation of the effect of the intervention (9). The same team of observers were used for the baseline and endline structured observations and this may have introduced observer bias during the endline data collection.

The study design, methodology and sample size limits the generalisability of our findings to the study HCFs and particularly to women with uncomplicated vaginal births in the facility. Furthermore, the non-randomisation of the facilities means that unobserved confounders may have had an effect on outcomes. We also cannot say if our intervention led to sustained adoption of the desired behaviours and a longer observation period would allow the exploration of the sustained adoption of behavioural outcomes.

As this is a limited efficacy/proof of concept study, it was not appropriate to measure maternal or newborn health related outcomes. Future larger-scale trials using measures of health impact are encouraged to determine whether the observed increases in handwashing with soap are sufficient to reduce infection.

\section{Conclusion}

The study suggests the potential of a multi modal behaviour change intervention to improve hygiene behaviours specifically hand hygiene- linked to maternal and neonatal infection during labour, delivery and post-natal care. More rigorous and larger scale studies are warranted to inform practice and policy change recommendations related to maternal and neonatal infection prevention.

\section{Abbreviations}

LMIC - Low and Middle income countries

PNC - Postnatal care

LD - Labour and Delivery

HCF - Healthcare facility

PHC - Primary Health Centres

BCD - Behaviour Centred Design

ANC - Antenatal Care

WASH - Water, Sanitation and Hygiene

OR - Odds Ratio

AOR - Adjusted Odds Ratio

Cl - Confidence Interval

\section{Declarations}


Data availability statement: The protocols, datasets used and/or analysed during this study are available from the corresponding author upon reasonable request. All data relevant to the study are included in the article or uploaded as supplementary information.

Ethics approval and consent to participate: The study was conducted according to the guidelines of the Declaration of Helsinki, and approved by the Institutional Review Board at the London School of Hygiene and Tropical Medicine (Reference 16128, 21 November 2018, 22 May 2019-Amendment \& Reference 19103, 04 March 2020, 01 May 2020 - Amendment) and the Cambodia National Ethics Committee for Health research (NECHR Number 13, 28/01/2019, NECHR Number 134, 24 May 2019-Amendment, NEHCR Number 131, 15 June 2020). All participants provided written informed consent prior to participation.

Consent for publication: Not required.

Competing interests: All authors have completed the ICMJE uniform disclosure form at www.icmje.org/coi_disclosure.pdf and declare: no support from any organisation for the submitted work; no financial relationships with any organisations that might have an interest in the submitted work in the previous three years; no other relationships or activities that could appear to have influenced the submitted work.

Funding: This research was funded by the Australian Government's Department of Foreign Affairs and Trade (DFAT) under Water for Women fund, grant number WRA089. The views and opinions expressed therein are those of the authors and do not necessarily reflect those of the funding organisations or affiliated institutions. The funder played no role in the study design, in the collection, analysis or interpretation of the data, in the writing of the paper or in the decision to submit the article for publication. All authors were independent from the funders.

Authors' contributions: Conceptualisation, A.M., C.S., P.I. and R.D.; methodology, H.B. and R.D.; formal analysis, P.P. and Y.N.; investigation, H.B., P.P., S.L. and Y.N.; resources, C.S., P.I., and S.L.; data curation, P.P. and Y.N.; writingoriginal draft preparation, Y.N.; writing-review and editing, A.M., C.S., H.B., P.I., P.P., R.D., S.L. and Y.N.; supervision, R.D.; project administration, Y.N. and C.S.; funding acquisition, C.S. and R.D. All authors have read and agreed to the published version of the manuscript. All authors had full access to all of the study data and take responsibility for the integrity of the data and the accuracy of the data.

Acknowledgments: We would like to give a very special thanks to the women who agreed to participate in this study without benefit to themselves as well as the staff and management of the participating HCF. Special thanks go to the following institutions and individuals for their participation and support: $\mathrm{MoH}-\mathrm{Cambodia}$, the National Institute of Public Health (NIPH), the National Centre for Maternal and Child Health, the Provincial Health Department and Operational District-Kampong Chhnang, WaterAid Cambodia, WaterAid Australia and other development partners. A very special thanks to WaterAid Cambodia and NIPH team for coordinating the study, and the special contributions of Saray Vannat, Bouth Seyha, Dip Bopha, Hem Navy, OI Sokhoeun, Ul Pisey, and Vouchnea Tang. All views expressed in this manuscript are those of the authors and do not necessarily reflect those of the funding organisations or affiliated institutions.

\section{References}

1. Fleischmann-Struzek, C. et al. The global burden of paediatric and neonatal sepsis: a systematic review. Lancet Respir Med, 6 (3), 223-230 (2018). 
2. Say, L. et al. Global causes of maternal death: a WHO systematic analysis. Lancet Glob Health, 2 (6), e323-33 (2014).

3. WHO. A guide to the implementation of the WHO multimodal hand hygiene improvement strategy. 2009. Available at: whqlibdoc who int/hq/2009/WHO_IER_PSP_2009 02_eng pdf. 2011.

4. WHO, UNICEF, UNFPA. Pregnancy, childbirth, postpartum, and newborn care: a guide for essential practice (World Health Organization, 2003).

5. World Health Organization. Every Newborn: an action plan to end preventable deaths. 2014. Geneva: World Health Organization. 2019:1-55.

6. Blencowe, H. et al. Clean birth and postnatal care practices to reduce neonatal deaths from sepsis and tetanus: a systematic review and Delphi estimation of mortality effect. BMC Public Health, 11 (Suppl 3), S11 (2011).

7. Curtis, V. A., Danquah, L. O. \& Aunger, R. V. Planned, motivated and habitual hygiene behaviour: an eleven country review. Health Educ Res, 24 (4), 655-673 (2009).

8. Parveen, S. et al. Barriers to and motivators of handwashing behavior among mothers of neonates in rural Bangladesh. BMC Public Health, 18 (1), 483 (2018).

9. Ram, P. K. et al. Is structured observation a valid technique to measure handwashing behavior? Use of acceleration sensors embedded in soap to assess reactivity to structured observation. Am J Trop Med Hyg, 83 (5), 1070-1076 (2010).

10. de Graft-Johnson, J. et al. The maternal, newborn and child health continuum of care. Opportunities for Africa's Newborns. 2006:23-36.

11. Kerber, K. J. et al. Continuum of care for maternal, newborn, and child health: from slogan to service delivery. The Lancet, 370 (9595), 1358-1369 (2007).

12. Kikuchi, K. et al. Effective Linkages of Continuum of Care for Improving Neonatal, Perinatal, and Maternal Mortality: A Systematic Review and Meta-Analysis. PLOS ONE, 10 (9), e0139288 (2015).

13. Gon, G., de Barra, M., Dansero, L., Nash, S. \& Campbell, O. M. Birth attendants' hand hygiene compliance in healthcare facilities in low and middle-income countries: a systematic review. BMC health services research, 20 (1), 1-16 (2020).

14. Buxton, H. et al. Hygiene During Childbirth: An Observational Study to Understand Infection Risk in Healthcare Facilities in Kogi and Ebonyi States, Nigeria. Int J Environ Res Public Health. 2019;16(7).

15. Nalule, Y. et al. Hygiene along the continuum of care in the early post-natal period: an observational study in Nigeria. BMC pregnancy and childbirth, 20 (1), 1-11 (2020).

16. Bazzano, A. N., Taub, L., Oberhelman, R. A. \& Var, C. Newborn Care in the Home and Health Facility: Formative Findings for Intervention Research in Cambodia. Healthcare (Basel), 4 (4), 94 (2016).

17. Gon, G. et al. Hands washing, glove use, and avoiding recontamination before aseptic procedures at birth: a multicenter time-and-motion study conducted in Zanzibar. American journal of infection control, 47 (2), $149-$ 156 (2019).

18. Nalule, Y. et al. Hand hygiene during facility-based childbirth in Cambodia: a theory-driven, mixed-methods observational study. BMC Pregnancy and Childbirth, 21 (1), 429 (2021).

19. Nalule, Y. et al. Hand Hygiene during the Early Neonatal Period: A Mixed-Methods Observational Study in Healthcare Facilities and Households in Rural Cambodia. International journal of environmental research and public health, 18 (9), 4416 (2021). 
20. Var, C. et al. A Linked Community and Health Facility Intervention to Improve Newborn Health in Cambodia: The NICCI Stepped-Wedge Cluster-Randomized Controlled Trial. International Journal of Environmental Research and Public Health, 17 (5), 1559 (2020).

21. Huttinger, A. et al. Evaluation of Membrane Ultrafiltration and Residual Chlorination as a Decentralized Water Treatment Strategy for Ten Rural Healthcare Facilities in Rwanda. International Journal of Environmental Research and Public Health, 12 (10), 13602-13623 (2015).

22. Mathai, E., Allegranzi, B., Kilpatrick, C. \& Pittet, D. Prevention and control of health care-associated infections through improved hand hygiene. Indian journal of medical microbiology, 28 (2), 100-106 (2010).

23. Danda, G., Dube, K., Dube, P., Mudokwenyu-Rawdon, C. \& Bedwell, C. An observational study of midwives' practices to prevent peripartum sepsis in Zimbabwe. African Journal of Midwifery and Women's Health, 9 (1), 17-21 (2015).

24. Greenland, K., Iradati, E., Ati, A., Maskoen, Y. Y. \& Aunger, R. The context and practice of handwashing among new mothers in Serang, Indonesia: a formative research study. BMC public health, 13 (1), 1-11 (2013).

25. Parveen, S. et al. Barriers to and motivators of handwashing behavior among mothers of neonates in rural Bangladesh. BMC public health, 18 (1), 483 (2018).

26. Ram, P. K. et al. Impact of an Intensive Perinatal Handwashing Promotion Intervention on Maternal Handwashing Behavior in the Neonatal Period: Findings from a Randomized Controlled Trial in Rural Bangladesh. Biomed Res Int, 2017, 6081470 (2017).

27. Rhee, V. et al. Maternal and birth attendant hand washing and neonatal mortality in southern Nepal. Arch Pediatr Adolesc Med, 162 (7), 603-608 (2008).

28. Park, J. Y., Pardosi, J. F. \& Seale, H. Examining the inclusion of patients and their family members in infection prevention and control policies and guidelines across Bangladesh, Indonesia, and South Korea. American journal of infection control, 48 (6), 599-608 (2020).

29. Horng, L. et al. Healthcare worker and family caregiver hand hygiene in Bangladeshi healthcare facilities: results from the Bangladesh National Hygiene Baseline Survey. Journal of Hospital Infection, 94 (3), 286-294 (2016).

30. Aunger, R. \& Curtis, V. Behaviour Centred Design: towards an applied science of behaviour change. Health Psychol Rev, 10 (4), 425-446 (2016).

31. Buxton, H. et al. Barriers and opportunities experienced by staff when implementing infection prevention and control guidelines during labour and delivery in healthcare facilities in Nigeria. J Hosp Infect, 103 (4), 428-434 (2019).

32. Bazzano, A. N., Oberhelman, R. A., Potts, K. S., Gordon, A. \& Var, C. Environmental factors and WASH practices in the perinatal period in Cambodia: implications for newborn health. Int J Environ Res Public Health, 12 (3), 2392-2410 (2015).

33. White, S., Thorseth, A. H., Dreibelbis, R. \& Curtis, V. The determinants of handwashing behaviour in domestic settings: An integrative systematic review. Int J Hyg Environ Health, 227, 113512 (2020).

34. Gaube, S., Fischer, P. \& Lermer, E. Hand(y) hygiene insights: Applying three theoretical models to investigate hospital patients' and visitors' hand hygiene behavior. PLoS One, 16 (1), e0245543 (2021).

35. Gon, G. et al. Behavioural Determinants of Hand Washing and Glove Recontamination before Aseptic Procedures at Birth: A Time-and-Motion Study and Survey in Zanzibar Labour Wards. International journal of environmental research and public health, 17 (4), 1438 (2020). 
36. de Barra, M. et al. Understanding infection prevention behaviour in maternity wards: A mixed-methods analysis of hand hygiene in Zanzibar. Soc. Sci. Med, 272, 113543 (2021).

37. Huis, A. et al. A systematic review of hand hygiene improvement strategies: a behavioural approach. Implementation Science, 7 (1), 92 (2012).

38. Smiddy, M. P. \& Creedon, R. O. C. SA. Systematic qualitative literature review of health care workers' compliance with hand hygiene guidelines. Am J Infect Control, 43 (3), 269-274 (2015).

39. Srigley, J. A. et al. Applying psychological frameworks of behaviour change to improve healthcare worker hand hygiene: a systematic review. J Hosp Infect, 91 (3), 202-210 (2015).

40. Naikoba, S. \& Hayward, A. The effectiveness of interventions aimed at increasing handwashing in healthcare workers - a systematic review. J Hosp Infect, 47 (3), 173-180 (2001).

41. Kuzu, N., Ozer, F., Aydemir, S., Yalcin, A. N. \& Zencir, M. Compliance with hand hygiene and glove use in a university-affiliated hospital. Infect Control Hosp Epidemiol, 26 (3), 312-315 (2005).

42. Pittet, D. Hand hygiene: improved standards and practice for hospital care. Curr Opin Infect Dis, 16 (4), $327-$ 335 (2003).

43. Erasmus, V. et al. Systematic review of studies on compliance with hand hygiene guidelines in hospital care. Infect Control Hosp Epidemiol, 31 (3), 283-294 (2010).

44. O'Boyle, C. A., Henly, S. J. \& Larson, E. Understanding adherence to hand hygiene recommendations: the theory of planned behavior. Am J Infect Control, 29 (6), 352-360 (2001).

45. WHO. WHO guidelines on hand hygiene in health care: First Global Patient Safety Challenge: Clean Care is Safer Care (World Health Organization, Geneva, 2009).

46. Puchalski Ritchie, L. M. et al. Low- and middle-income countries face many common barriers to implementation of maternal health evidence products. Journal of Clinical Epidemiology, 76, 229-237 (2016).

47. Hor S-y, Hooker C, ledema R, Wyer M, Gilbert GL, Jorm C, et al. Beyond hand hygiene: a qualitative study of the everyday work of preventing cross-contamination on hospital wards. BMJ Quality \&amp;amp; Safety. 2017;26(7):552.

48. Loftus, R. W. et al. Hand contamination of anesthesia providers is an important risk factor for intraoperative bacterial transmission. Anesth Analg, 112 (1), 98-105 (2011).

49. Wilson, J. et al. The misuse and overuse of non-sterile gloves: application of an audit tool to define the problem. J Infect Prev, 16 (1), 24-31 (2015).

50. Cronin, W. A., Quansah, M. G. \& Larson, E. Obstetric infection control in a developing country. J Obstet Gynecol Neonatal Nurs, 22 (2), 137-144 (1993).

51. Gon, G., Dancer, S., Dreibelbis, R., Graham, W. J. \& Kilpatrick, C. Reducing hand recontamination of healthcare workers during COVID-19. Infection control and hospital epidemiology, 41 (7), 870-871 (2020).

52. Sutton, R. M. et al. Low-dose, high-frequency CPR training improves skill retention of in-hospital pediatric providers., 128 (1), e145-e51 (2011).

53. Evans, C. L. et al. Peer-assisted learning after onsite, low-dose, high-frequency training and practice on simulators to prevent and treat postpartum hemorrhage and neonatal asphyxia: A pragmatic trial in 12 districts in Uganda. PLoS One, 13 (12), e0207909 (2018).

54. Gomez, P. P. et al. Accelerating newborn survival in Ghana through a low-dose, high-frequency health worker training approach: a cluster randomized trial. BMC Pregnancy and Childbirth, 18 (1), 72 (2018). 
55. Ugwa, E. et al. Simulation-based low-dose, high-frequency plus mobile mentoring versus traditional groupbased trainings among health workers on day of birth care in Nigeria; a cluster randomized controlled trial. BMC Health Services Research, 20 (1), 586 (2020).

56. Halder, A. K., Molyneaux, J. W., Luby, S. P. \& Ram, P. K. Impact of duration of structured observations on measurement of handwashing behavior at critical times. BMC Public Health, 13 (1), 705 (2013).

57. George, C. M. et al. Randomized controlled trial of hospital-based hygiene and water treatment intervention (CHoBI7) to reduce cholera. Emerging infectious diseases, 22 (2), 233 (2016).

58. Loharikar, A. et al. Long-term impact of integration of household water treatment and hygiene promotion with antenatal services on maternal water treatment and hygiene practices in Malawi. The American journal of tropical medicine and hygiene, 88 (2), 267-274 (2013).

59. Briere, E. C. et al. Impact of integration of hygiene kit distribution with routine immunizations on infant vaccine coverage and water treatment and handwashing practices of Kenyan mothers. Journal of Infectious Diseases, 205 (suppl_1), S56-S64 (2012).

60. Parker, A. A. et al. Sustained high levels of stored drinking water treatment and retention of hand-washing knowledge in rural Kenyan households following a clinic-based intervention. Epidemiology \& Infection, 134 (5), 1029-1036 (2006).

61. McBride, C. M., Emmons, K. M. \& Lipkus, I. M. Understanding the potential of teachable moments: the case of smoking cessation. Health education research, 18 (2), 156-170 (2003).

62. Ogden, J. \& Hills, L. Understanding sustained behavior change: the role of life crises and the process of reinvention., 12 (4), 419-437 (2008).

63. Phelan, S. Pregnancy: a "teachable moment" for weight control and obesity prevention. Am J Obstet Gynecol, 202 (2), 135 (2010). .e1-.e1358

64. Joy, E. A., Van Hala, S. \& Marshall, E. Pregnancy as an opportunity for behavior change. Current sports medicine reports, 3 (6), 327-329 (2004).

\section{Supplementary Files}

This is a list of supplementary files associated with this preprint. Click to download.

- Nalule.et.al.SRSupplementarytables14.pdf 